\title{
Assessing the Use of Communication Robots for Recreational Activities at Nursing Homes
}

\author{
Noriaki Kuwahara \\ Kyoto Institute of Technology \\ 1 Hashigami-cho Matsugasaki \\ Sakyo-ku, Kyoto city Kyoto, Japan \\ +81-75-724-7494 \\ nkuwahar@kit.ac.jp
}

\begin{abstract}
We are using information communication technology and communication robots (hereafter referred to as "robots") to develop a service to assist recreational activities at nursing homes. The service relies on visual content to deliver a variety of recreational activities, from exercises to reminiscence therapy. Robots support those activities by interacting with nursing home residents. These services are currently being evaluated at various elderly care facilities, where they have been installed. This report will review the recreational contents that are currently under development, the outline of the assessments, and present the latest data from those assessments.
\end{abstract}

\section{Categories and Subject Descriptors}

I.2.9 [Artificial Intelligence]: Robotics - Commercial robots and applications.

\section{General Terms}

Design, Experimentation.

\section{Keywords}

Communication Robot, Aging, Care, Recreation.

\section{INTRODUCTION}

Recreational activities at elderly care facilities play an essential role in the maintenance of a quality life. Recreation serves more than the purpose of bringing enjoyment to the residents; it also helps with rehabilitation $[1,2]$. On occasion, care givers get stuck in a rut, and the less experienced staff in particular tends to resist taking the lead in livening things up. With regards to managing recreational activities, one of the issues that needs addressing is the training of the young staff [3]. Because of this, nursing facilities frequently end up using DVDs or visual aid in leading exercises, singing and other activities. Although healthy elderly possibly enjoy such visual contents, it is difficult to sustain the focus of elderly dementia patients on visual contents. We set out to evaluate the efficacy of the robot services we developed to augment visual contents used at elderly care facilities.

Reports indicate that replacing message boards and other static forms of communication with robots that communicate improves the message's reliability. Other reports have shown that when robots facilitated face-to-face communication between two elderly dementia patients through the TV phone, the subjects were more likely to direct their gaze toward the TV monitor and liven up conversations [4, 5]. The presence of robots is therefore expected to increase one's gaze and focus on the visual contents. It is anticipated that the addition of robots opens up the usual staff-to-residents communication to a three-way channel that creates more opportunities for communication between the care staff and the residents. As a result, expectations are that the services currently under development will reduce stress on care givers who manage recreational activities, and that will in turn bring about positive changes to the entire recreation program.

Nippon Telegraph and Telephone West Corporation (hereafter referred to as "NTT West") and Nippon Telegraph and Telephone East Corporation (hereafter referred to as "NTT East") are currently developing the recreation service in the aforementioned system using information communication technology and communication robots. The recipients of the system are two nursing facilities in the Kansai region and two in the Kanto region. They are either in the midst of or preparing for an assessment. This report shows the results of just one part of an assessment that took place in a Kansai nursing facility. With regards to the impact that the service has had on the quality of nursing care, the results shown were evaluated through Dementia Care Mapping [6] (hereafter referred to as DCM).

\section{METHOD}

\subsection{System Outline}

Fig. 1 shows the outline of the system. The hardware used is Hikari $\mathrm{Box}^{+}$[7], a set-top box provided by NTT West, and a robot connected to that. We used FLET'S Hikari [8], an Internet service provided by NTT West and NTT East. Nursing home recreational activities (visual contents) are available as application of Hikari $\mathrm{Box}^{+}$. Hikari $\mathrm{Box}^{+}$and robot are connected via wireless LAN, and the robot synchronizes its actions with the visual content. The robot Sota [9], as seen in the drawing, appears only as a torso, 30 centimeters in height and used on a table top. Hikari Box ${ }^{+}$is shaped like a box measuring $115 \mathrm{~mm} \times 105 \mathrm{~mm} \times 31.5 \mathrm{~mm}$ and operated with remote control buttons. The TV is connected through an HDMI cable. Visual contents vary from children's stories and exercises, to quizzes (calculations, kanji characters), reminiscing (topics from the past), and an introduction to famous local spots. 


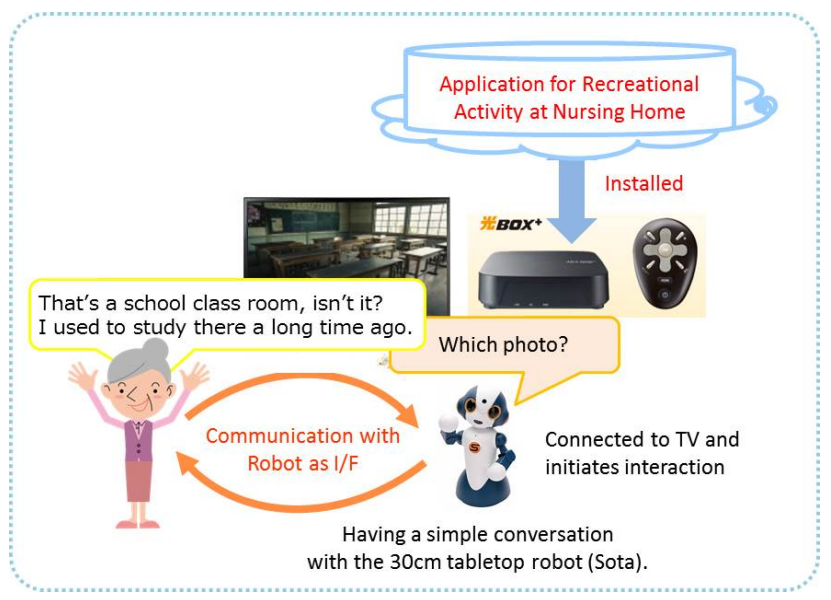

Figure 1. System Outline

The nursing home recreation services can be activated with the mere press of the remote control button on Hikari $\mathrm{Box}^{+}$. On the other hand, after the remote controller is used at the time of starting recreation activities, moving on to the next question on a quiz or etc. the robot begins to operate. The robot then gesticulates and speaks words of starting recreations or encouragement. If the recreational content has not been activated, it looks around the room to identify faces, then turns in the general direction of the faces and speak. However, the robot does not possess the intelligence to understand dialogue initiated by the residents. It is equipped with just the very basic function of acknowledging a statement at the appropriate time, or some other superficial responses.

\subsection{Assessment Schedule}

Care staff were given a 2-week pretrial period to become accustomed to the Hikiari $\mathrm{Box}^{+}$and learnt how to operate the nursing recreation system. At the same time, contents were narrowed down to those that will undergo the assessment. Following that, during a 3-month trial period, a portion of the usual recreational programs carried out at the facilities incorporated Hikari $\mathrm{Box}^{+}$and robot system. Pretrials began in mid-June for the Kansai region's assessment, and a 3-month trial has been underway since July. The assessment schedule for the Kanto region is yet to be announced.

\subsection{Assessment Methods}

\subsubsection{Study Participants}

The assessments, as mentioned before, are taking place in two facilities in the Kansai, and will take place in the Kanto regions. For this report, results of the first DCM administered at an elderly nursing home in Kyoto -- the only ones available at the time of this report's writing -- will be presented. The following is the profiles of the three elderly dementia patients who took part in the assessment:

- ID: A, Gender: Female, Age: 91, Disturbance of ADL: Severe, Dementia severity: Moderate

- ID: B, Gender: Female, Age: 88, Disturbance of ADL: Severe, Dementia severity: Severe

- $\quad$ ID: C, Gender: Female, Age: 81 , Disturbance of ADL: Moderate, Dementia severity: Severe

\subsubsection{Assessment Steps}

The assessment included a survey after the 2 -week pretrial period that asked the subjects their impressions and opinions of each recreational activity. Then, every two weeks during the 3-month trial period that followed, the GBS scale [10] for rating the severity of dementia and its qualitative differences are being used to measure the subjects' emotional function and mental state. Furthermore, on the third month when the nursing staff and the residents have become fully accustomed to the robot and the recreational activities, two evaluations by DCM take place to assess changes in the quality of the facilities' nursing care caused by the incorporation of the new service. Results for this report were extracted from the Kyoto facility, aforementioned, as they were the only one available at the time of this writing. The following is a brief summary about DCM.

DCM is a method that evaluates person-centered care [11]. Person-centered care is based on a theory for dementia care developed by the late Professor of psychology at Bradford University, Tom Kitwood. DCM is based on observing a dementia patient for over 6 hours in a day. Every five minutes, the patient's activities are codified in a Behavior Category Code; from there, the patient's state is further categorized into one of six Mood Engagement levels $(+5,+3,+1,-1,-3,-5)$, which correspond to the highest level (well-being) to the lowest (illbeing). BCC stratifies the patients' verbal and nonverbal interactions with their surroundings into 24 types, from A through Z. DCM is carried out by specially certified individuals called mappers. The mapping is typically performed by three such individuals, who after a period of observation come up with a consensus on $\mathrm{BCC}$ and $\mathrm{ME}$ values before arriving at a final diagnosis.

In order to observe changes in the residents participating in recreational activities, we kept the observation time to two hours, which included group recreation time and the residents' free time. Three certified mappers (two men, one woman) performed the evaluation.

\subsubsection{Carrying out Nursing Recreation Activities}

For the privately run nursing home in Kyoto, the mappers observed the residents on September 7, from 2-4 pm, recreation time. The timetable for that day was as follows:
I. $14: 00 \sim 14: 10 \quad$ TV viewing without robot
II. 14:10 14:50 Recreation with robot (kanji quiz, math quiz, Kyoto shrine visits)
III. 14:50 15:20 TV viewing without robot, with tea
IV. 15:20 15:35 Tea break with stand-alone robot
V. 15:35 15:45 Tea break and TV viewing without robot
VI. 15:45 16:00 Recreation with robot (children's stories)

Figure 2 shows the layout of the room in which the recreation assessment took place.

Assessments usually take place at the facilities' group recreation time, other residents are present. All the residents, including the participants, are in wheelchairs. The mappers watched the participants from where they could not be seen. 


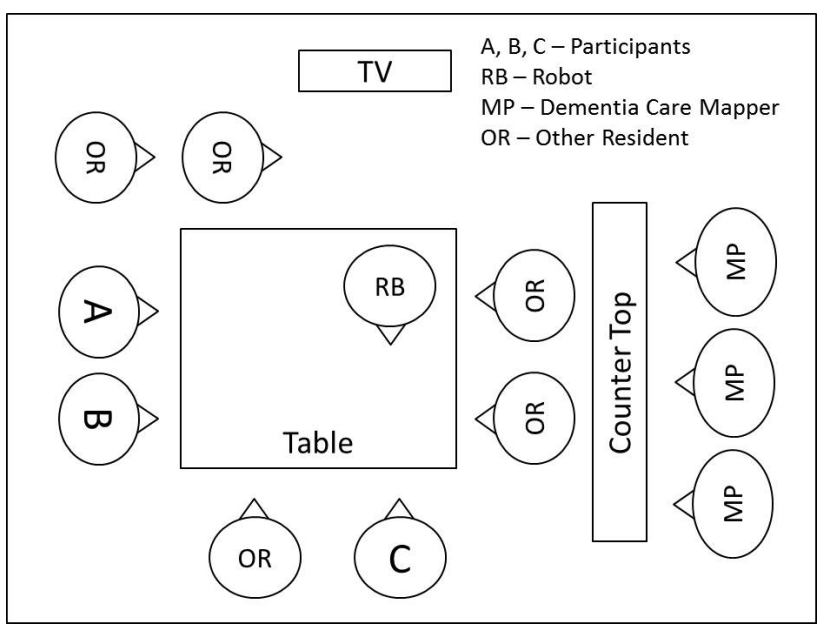

Figure 2. Room Layout during Assessment

\section{RESULT}

\subsection{Transitions in BCC and ME Values over Time}

Figs. 3-5 show the changes in the $\mathrm{BCC}$ and $\mathrm{ME}$ numbers over time for each participant. I-IV in the figure refer to the recreation timetable mentioned in the section 2.3.3. The numbers 1-7 refer to the amount of time the residents spent in recreation using the robot or with the robot. As previously mentioned, the observation time was between 2 and $4 \mathrm{pm}$, each code applying to 5-minute intervals. Participant B did not attend from 14:00-14:10. There is no code between then.

Within the time frame that they were in recreation or interacting with robots, the participants were primarily engaged in one of the following: E - creating or expressing one's self, $\mathrm{G}$ - reminiscing, looking back on one's life, I - activities that involve one's intellectual capacities, L - activities that bring about enjoyment, feelings of leisureliness, $\mathrm{O}$ - activities with objects (the robot), showing attachment to or obsessing over objects (the robot).

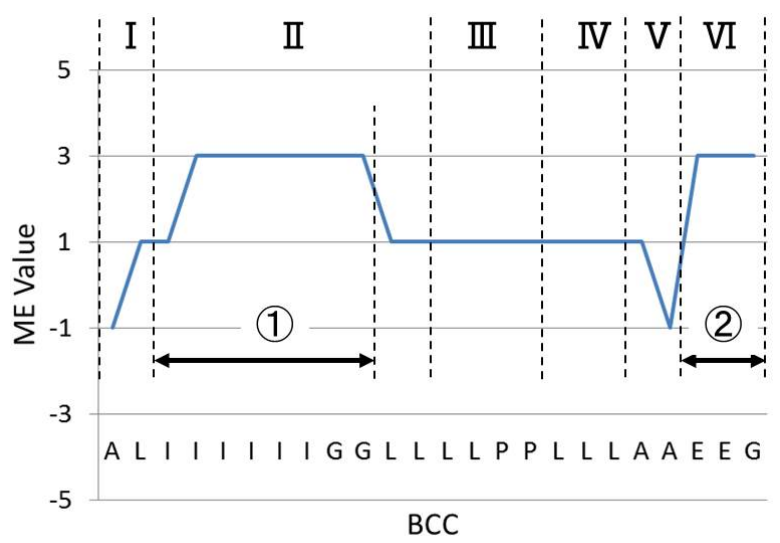

Figure 3. Participant A's BCC and ME value changes

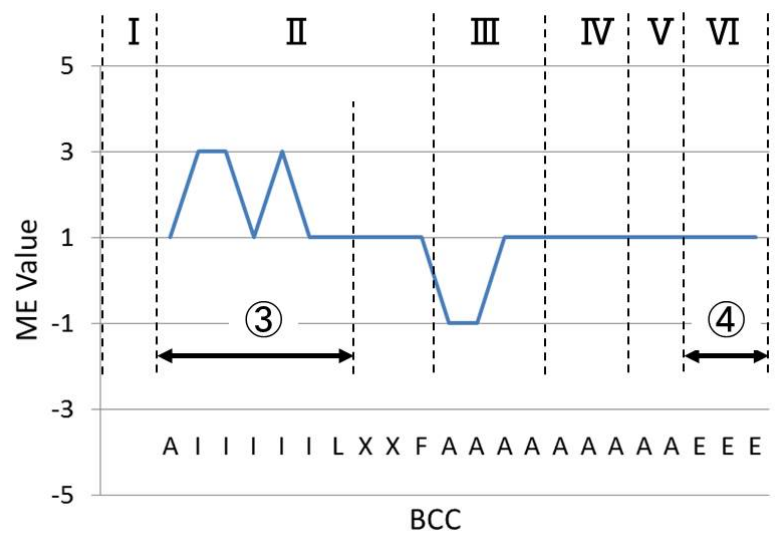

Figure 4. Participant B's BCC and ME value changes

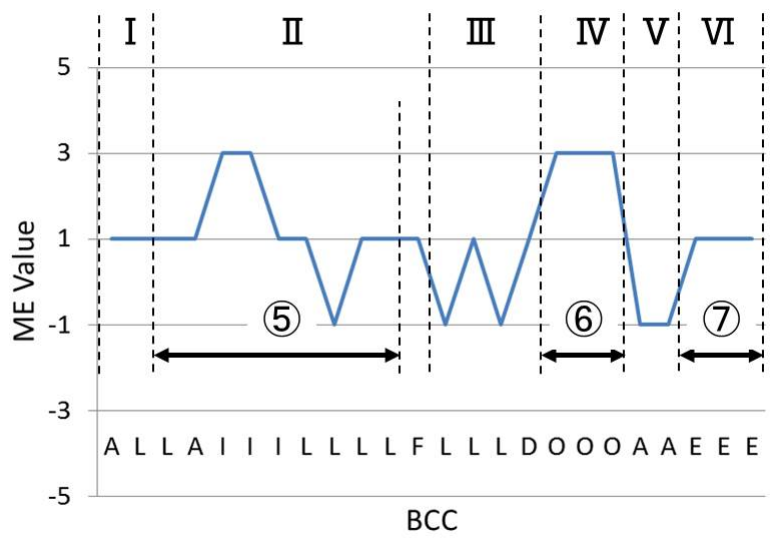

Figure 5. Participant C's BCC and ME value changes

On the other hand, they were engaged in one of the following when outside of this time frame: A - interacting verbally or nonverbally with those around them, $\mathrm{P}$ - receiving some form of physical care, $\mathrm{X}$ - activities related to elimination.

\subsection{Interactions through Recreation and Robots, and Other Comparisons}

In the next phase, we compared the ME averages extracted from the time frames 1 through 7 when the residents interacted through recreation and with the robot seen in Figs. 3-5 and outside of those time frames. The results are shown in Fig. 6. The average ME values for all three participants during the times they spent in recreation or with the robot were considerably higher than those for the time spent outside of those time frames.

\section{CONCLUSION}

Figures 3 to 5 show that the three participants were fully engaged during their time in recreation with robot or interacting with the robot, whether it was in creative or expressive, intellectual or leisurely activities. As seen in Fig. 6, the average ME values within that time frame were notably higher than those outside of that time frame. This is to say, the service has a strong potential to contribute positively to a better quality of life for residents with dementia. 


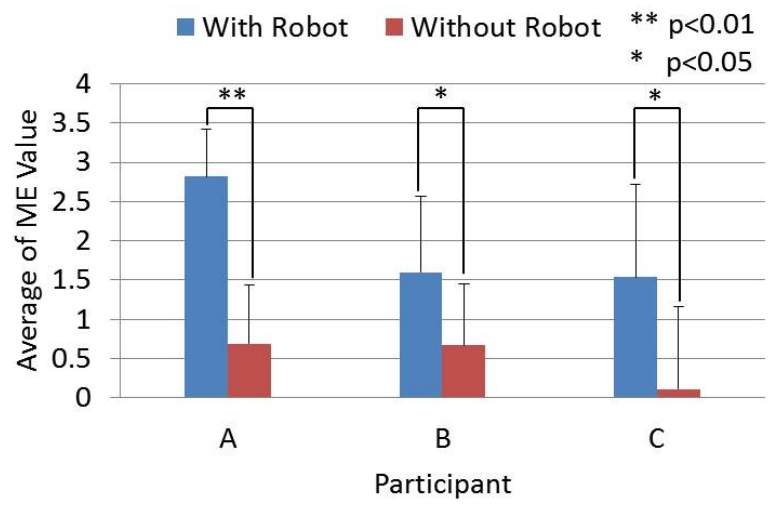

Figure 6. Comparison of average ME values

The notable event that suggests the usability of incorporating robots as a part of the service was found. It is worth noting participant C's response when the robot was activated separately from the recreational activities, which corresponds to number 6 in fig. 5. During the TV viewing time of phase III when she became restless, the robot was activated and she kept it all to herself. That triggered communication between the two and she regained her composure. This example shows that even with extremely limited interactive functions, the robot has the potential to stabilize the dementia patient's mood. During this time, coincidentally, the robot made sounds and gestures that timed perfectly with the pauses in participant C's speech. As a result, we believe that even a small improvement in the interactive functions of the robot in a standalone situation will greatly increase the value of its presence in care facilities. We would like to further assess the usefulness of this service by analyzing data extracted from other facilities.

\section{ACKNOWLEDGMENTS}

We would like to thank NTT West and NTT East for their help in this research -- namely coordinating the assessment and for providing the equipment. We also thank SUPER COURT Co., Ltd., the privately-run retirement home for participating in the study, and the DCM Kansai region block association for assisting us in the DCM. And a part of this study was supported by JSPS KANENHI Grant Number 15H01698.

\section{REFERENCES}

[1] Doi, T., Kuwahara, N., and Morimoto, K. 2015. Effective Design of Traditional Japanese Tea Ceremony in a Group Home for the Elderly with Dementia. Springer LNCS 9184, 413-422. DOI=http://link.springer.com/chapter/10.1007/9783-319-21070-4 41 .

[2] Ikenobo, Y., Mochizuki, Y., and Kuwahara, N. 2015. Usefulness of Ikebana a Nursing Care Environment. Springer LNCS 9185, 441-447. DOI=http://link.springer.com/chapter/ 10.1007/978-3-319-21070-4 44.

[3] Yamamoto, A. et.al. 2015. The Transfer of Expertise in Conducting a Participatory Music Therapy during a Combined Rehabilitation-Recreational Program in an Elderly Care Facility. Springer LNCS 9184, 500-511.

DOI=http://link.springer.com/chapter/10.1007/978-3-31921070-4 51 .

[4] Yonezawa, T., Yamazoe, H., Utsumi, A., and Abe, S. 2013 Attractive, Informative, and Communicative Robot System on Guide Plate as an Attendant with Awareness of User's Gaze. Paladyn. Journal of Behavioral Robotics, 4, 2, 113-122.

[5] Yonezawa, T., Yamazoe, H., Utsumi, A., and Abe, S. 2011 Assisting video communication by an intermediating robot system corresponding to each user's attitude. Human Interface Society Journal, 13, 3, 5-13.

[6] Fossey, J., Lee, L., and Ballard, 2002. C. Dementia care mapping as a research tool for measuring quality of life in care settings: psychometric properties. International Journal of Geriatric Psychiatry, 17, 11, 1064-1070.

[7] https://www.ntt-west.co.jp/kiki/hikaribox/spec/ (in Japanese)

[8] http://hikari-n.jp/west/service/ (in Japanese)

[9] https://www.vstone.co.jp/products/sota/ (in Japanese)

[10] Bråne G. The GBS-scale - a Geriatric Rating Scale - and its Clinical Application. 1989, Gothenburg University, Gothenburg, Sweden.

[11] Kitwood, T., 1999, When your heart wants to remember: person-centred dementia care. In RCN Nursing Update, Nursing Standard, 13. 1-22. 